\title{
A NOTE ON WAVE PROPAGATION IN BONE
}

In a recent work on wave propagation, Ambardar and Ferris [1] modelled long bone as a two-layered cylindrical structure, consisting of a thin inner layer of spongy material and a thick outer layer of more compact bone. Both types of bone were taken to be transversely isotropic and piezoelectric effects were included. The authors gave detailed information on frequency spectra, but no numerical data, or data on transients, were presented. Here an explicit algebraic, long wavelength approximation to the lowest mode of compressional wave propagation in an infinite two-layered cylinder, the layers being transversely isotropic, is given. Piezoelectric effects are not included, but imperfect layer bonding is allowed. Information on transients is also given.

The work is a direct extension of a previous paper by the author [2] in which a pressure shock problem for a cylinder made of isotropic layers was considered. In fact the solution procedures, and the form of the solution, are identical to those in reference [2]. Differences arise only through the structure of the parameters and only that detail will be presented here.

For the pressure shock problem specified in reference [2], the solutions now are

$$
\begin{gathered}
\frac{c_{12} e_{r r}}{\sigma_{0}}=\frac{R_{h}\left[\left(\gamma R_{h}^{2} / \xi^{\prime 2}\right)-\delta / \beta\right]}{U_{1}\left[1+R_{\rho}\left(R_{h}-1\right)\right]}\left[\frac{1}{3}+\int_{0}^{\psi^{\prime}} \mathrm{Ai}(-\eta) \mathrm{d} \eta\right], \\
\frac{c_{11} e_{z z}}{\sigma_{0}}=\frac{c_{12} e_{z z}}{\sigma_{0}}=-\frac{R_{h}}{U_{1}\left[1+R_{\rho}\left(R_{h}-1\right)\right]}\left[\frac{1}{3}+\int_{0}^{\psi^{\prime}} \mathrm{Ai}(-\eta) \mathrm{d} \eta\right],
\end{gathered}
$$

where Ai denotes the Airy function and

$$
\psi^{\prime}=\left(\frac{2 R_{\rho} \sqrt{U_{1}}}{3 U_{2}}\right)^{1 / 3} \frac{\left(\sqrt{U_{1}} \tau-\zeta^{\prime}\right)}{\tau^{1 / 3}}, \quad \tau=\sqrt{\frac{c_{1}}{\rho_{1}}} \frac{t}{b}, \quad \zeta^{\prime}=\frac{z}{b}, \quad \xi^{\prime}=\frac{r}{b} .
$$

Further, following the sequence in reference [2], one has

$$
\begin{gathered}
\rho_{1} P_{1}=c_{1} U_{1}, \quad \rho_{2} P_{2}=c_{1} b^{2} U_{2}, \\
U_{1}=4 \nu-\frac{2 b_{1}}{c_{1}} \zeta+\frac{d_{1}}{c_{1}}, \quad \nu=\frac{\zeta}{2}+\frac{R_{\mu}\left(1-R_{h}^{2}\right) \delta}{2 \beta}-R_{\mu}\left(R_{h}^{2}-1\right) \theta, \\
{\left[2\left(\frac{a_{1}-e_{1}}{c_{1}}\right)-D_{B 1}\right] \zeta=D_{B 1}\left[1+R_{h}^{2}\left(\frac{a_{2}-e_{2}}{e_{2}}\right)\right] \frac{\delta}{\beta}+\frac{b_{1}-c_{1}}{c_{1}}+\frac{D_{B 1} R_{h}^{2}}{2}\left(\frac{b_{2}-c_{2}}{e_{2}}\right),} \\
R_{\mu}=c_{2} / c_{1}, \quad R_{h}=c / b, \\
\beta=\left(\frac{a_{2}-e_{2}}{c_{2}}\right)\left(R_{h}^{2}-1\right)\left[2\left(\frac{a_{1}-e_{1}}{c_{1}}\right)-D_{B 1}\right]-\frac{D_{B 1}}{R_{\mu}}\left(\frac{a_{1}-e_{1}}{c_{1}}\right)\left[1+\left(\frac{a_{2}-e_{2}}{e_{2}}\right) R_{h}^{2}\right], \\
\delta=\frac{1}{2}\left[2\left(\frac{a_{1}-e_{1}}{c_{1}}\right)-D_{B 1}\right]\left[\left(\frac{b_{2}-c_{2}}{c_{2}}\right)\left(1-R_{h}^{2}\right)-\left(\frac{b_{1}-c_{1}}{c_{1}}\right) \frac{1}{R_{\mu}}\right] \\
+\frac{1}{R_{\mu}}\left(\frac{a_{1}-e_{1}}{c_{1}}\right)\left[\frac{b_{1}-c_{1}}{c_{1}}+\frac{D_{B 1} R_{h}^{2}}{2}\left(\frac{b_{2}-c_{2}}{e_{2}}\right)\right],
\end{gathered}
$$




$$
\begin{aligned}
& \theta=\left\{2 R_{\rho}\left[\zeta+R_{\mu}\left(1-R_{h}^{2}\right)(\delta / \beta)\right]-\eta\right\}\left\{4 R_{\mu}\left[1+R_{\rho}\left(R_{h}^{2}-1\right)\right]\right\}^{-1}, \\
& D_{B 1}=\alpha_{1} b / c_{1}, \quad R_{\rho}=\rho_{2} / \rho_{1}, \\
& \eta=\left(d_{2} / c_{1}\right)-\left(d_{1} / c_{1}\right) R_{\rho}+\left(2 b_{2} \delta / c_{1} \beta\right)+\left(2 b_{1} R_{\rho} \zeta / c_{1}\right), \\
& U_{2}=U_{21}+U_{22} \\
& U_{21}=\left\{\left(V_{1}+V_{2}+V_{3}+V_{4}+V_{5}+V_{6}\right) /\left[1-R_{p}\left(1-R_{h}^{2}\right)\right]\right\}-4 R_{\mu} R_{h}^{2} \nu_{1} \\
& +2 R_{\mu} b_{2}\left(\xi_{2}+\xi_{3}+\xi_{4}+\xi_{5}\right) / c_{2} \xi_{1} \\
& \left.U_{22}=b_{2} R_{\mu} R_{h}^{2} \delta_{1} / c_{2}+\left\{\left(d_{2} / c_{2}\right) R_{\mu}-R_{\rho}\left[4 \nu-\left(2 b_{1} / c_{1}\right) \zeta+d_{1} / c_{1}\right)\right]\right\} \Psi, \\
& V_{1}=8 R_{\rho} R_{\mu}\left(1-R_{h}^{4}\right) \eta_{1}+2 R_{\rho} R_{\mu}\left(1-R_{h}^{4}\right) \beta_{1}-8 R_{\rho} \zeta_{1}, \\
& V_{2}=\left(2 R_{\mu} / \xi_{1}\right)\left(\xi_{2}+\xi_{3}+\xi_{4}+\xi_{5}\right)\left[R_{\rho}\left(1-R_{h}^{2}\right)-\left(b_{2} / c_{2}\right)\right]-2 R_{\rho} \Omega, \\
& \left.V_{3}=-2 R_{\mu} R_{h}^{2}\left[R_{\rho}\left(1-R_{h}^{2}-2 R_{h}^{2} \ln R_{h}\right)-2\right)\right] \nu_{1}, \\
& V_{4}=\frac{2 R_{p}\left(b_{1}-c_{1}\right)}{c_{1}}\left[\frac{c_{1} R_{\mu}\left(a_{2}-e_{2}\right)\left(1-R_{h}^{2}\right)\left(\xi_{2}+\xi_{3}+\xi_{4}+\xi_{5}\right)}{c_{2}\left(a_{1}-e_{1}\right) \xi_{1}}+\nabla_{1}+\nabla_{2}+\nabla_{3}+\nabla_{4}\right] \text {, } \\
& V_{5}=-R_{\mu}\left[2 R_{\rho} R_{h}^{2} \ln R_{h}+\left(b_{2} / c_{2}\right)\right] R_{h}^{2} \delta_{1}, \\
& V_{6}=2 R_{\rho}\left(\frac{b_{1}}{c_{1}} \zeta-2 \nu\right)\left[\frac{\phi}{2}-R_{\rho}\left(R_{h}-1\right) \Psi\right]-\left[\frac{d_{2}}{c_{2}} R_{\mu}-R_{\rho}\left(4 \nu-2 \frac{b_{1}}{c_{1}} \zeta+\frac{d_{1}}{c_{1}}\right)\right] \Psi \\
& \eta_{1}=\frac{\theta}{16}\left[\frac{R_{\rho}}{R_{\mu}}\left(4 \nu-\frac{2 b_{1}}{c_{1}} \zeta+\frac{d_{1}}{c_{1}}\right)-\frac{d_{2}}{c_{2}}\right]-\frac{b_{2}}{4 c_{2}} \beta_{1}, \\
& \beta_{1}=\frac{c_{2} \delta}{8 a_{2} \beta}\left[\frac{R_{\rho}}{R_{\mu}}\left(4 \nu-\frac{2 b_{1}}{c_{1}} \zeta+\frac{d_{1}}{c_{1}}\right)-1\right]-\frac{\theta b_{2}}{4 a_{2}}, \\
& \zeta_{1}=(\nu / 8)\left[2 \nu-\left(b_{1} / c_{1}\right) \zeta\right]-\left(b_{1} / 4 c_{1}\right) \Omega, \\
& \Omega=-\left(b_{1} / 4 a_{1}\right) \nu+\left(\zeta c_{1} / 8 a_{1}\right)\left[1-4 \nu+\left(2 b_{1} / c_{1}\right) \zeta-\left(d_{1} / c_{1}\right)\right], \\
& \xi_{1}=\frac{c_{1} R_{\mu}\left(1-R_{h}^{2}\right)\left(a_{2}-e_{2}\right)}{c_{2}\left(a_{1}-e_{1}\right)}\left[D_{B 1}-2\left(\frac{a_{1}-e_{1}}{c_{1}}\right)\right]-D_{B 1}\left[1+\left(\frac{a_{2}-e_{2}}{e_{2}}\right) R_{h}^{2}\right] \text {, } \\
& \xi_{2}=\left[2\left(\frac{2 a_{1}-e_{1}}{c_{1}}\right)-D_{B 1}\right] \Omega+\left(\frac{b_{1}-c_{1}}{c_{1}}\right) \nu+\left(\frac{b_{1}-c_{1}}{c_{1}}\right)\left[\frac{\phi}{2}-R_{\rho}\left(R_{h}-1\right) \Psi\right], \\
& \phi=\frac{1}{3} \zeta^{2}-\frac{2}{3} \nu-\frac{2}{3} R_{\rho}\left(R_{h}^{3}-1\right) \theta+2 R_{\rho} R_{h}^{2}\left[R_{h}\left(\ln R_{h}-1\right)+1\right] \xi \\
& +\frac{1}{3} R_{\rho}\left(R_{h}^{3}-1\right)\left(\delta^{2} / \beta^{2}\right)+R_{\rho} R_{h}^{3}\left(R_{h}-1\right) \gamma^{2}+2 R_{\rho}\left(R_{h}-1\right) R_{h}^{2} \delta \gamma / \beta, \\
& \xi=2 \theta+\gamma+\delta / \beta, \\
& \gamma=\left[\left(a_{2}-e_{2}\right) / e_{2}\right](\delta / \beta)+\left(b_{2}-c_{2}\right) / 2 e_{2} \text {, } \\
& \Psi=\left[\nu+\left(\zeta / D_{B 2}\right)+\frac{\phi}{2}-\theta-\frac{2 \nu}{D_{B 2}}\right]\left[1+R_{\rho}\left(R_{h}-1\right)\right]^{-1}, \quad D_{B 2}=\alpha_{2} b / c_{1}, \\
& \xi_{3}=\left[\frac{2\left(a_{1}-e_{1}\right)}{c_{1}}-D_{B 1}\right]\left(\nabla_{1}+\nabla_{2}+\nabla_{3}+\nabla_{4}\right)+D_{B 1}\left[1+\left(\frac{2 a_{2}-e_{2}}{e_{2}}\right) R_{h}^{4}\right] \beta_{1}, \\
& \nabla_{1}=-\frac{\left(b_{1}-c_{1}\right)}{2\left(a_{1}-e_{1}\right)}\left[\frac{\phi}{2}-R_{\rho}\left(R_{h}-1\right) \Psi\right]-\left(\frac{2 a_{1}-e_{1}}{a_{1}-e_{1}}\right) \Omega-\frac{\left(b_{1}-c_{1}\right) \nu}{2\left(a_{1}-e_{1}\right)},
\end{aligned}
$$




$$
\begin{gathered}
\nabla_{2}=\left(\frac{2 a_{2}-e_{2}}{c_{2}}\right)\left(\frac{c_{1}}{a_{1}-e_{1}}\right)\left(1-R_{h}^{4}\right) R_{\mu} \beta_{1}+\left(\frac{b_{2}-c_{2}}{2 c_{2}}\right)\left(\frac{c_{1}}{a_{1}-e_{1}}\right)\left(1-R_{h}^{4}\right) R_{\mu} \theta, \\
\nabla_{3}=R_{\mu}\left(\frac{c_{1}}{a_{1}-e_{1}}\right)\left[\frac{a_{2}}{2 c_{2}}\left(1-R_{h}^{2}\right)-\left(\frac{a_{2}-e_{2}}{c_{2}}\right) R_{h}^{2} \ln R_{h}\right] \delta_{1} R_{h}^{2}, \\
\delta_{1}=\frac{c_{2}}{2 a_{2}}\left[\frac{R_{\rho}}{R_{\mu}}\left(4 \nu-\frac{2 b_{1}}{c_{3}} \zeta+\frac{d_{1}}{c_{1}}\right)-1\right] \gamma+\frac{b_{2}}{2 a_{2}} \xi, \\
\nabla_{4}=R_{\mu}\left(\frac{b_{2}-c_{2}}{2 c_{2}}\right)\left(\frac{c_{1}}{a_{1}-e_{1}}\right)\left[\left(1-R_{h}^{2}\right) \Psi+\xi R_{h}^{4} \ln R_{h}\right], \\
\xi_{4}=\frac{\left(b_{2}-c_{2}\right) D_{B 1} R_{h}^{4} \theta}{2 e_{2}}+\frac{D_{B 1} R_{h}^{4}}{2}\left[\frac{a_{2}}{e_{2}}+\frac{2\left(a_{2}-e_{2}\right)}{e_{2}} \ln R_{h}\right] \delta_{1}, \\
\xi_{5}=\left[\left(b_{2}-c_{2}\right) / 2 e_{2}\right] R_{h}^{2} D_{B 1}\left[\Psi-R_{h}^{2} \xi \ln R_{h}\right], \\
\nu_{1}=\frac{1}{4} \xi\left\{\left(R_{\rho} / R_{\mu}\right)\left[4 \nu-\left(2 b_{1} / c_{1}\right) \zeta+d_{1} / c_{1}\right]-\left(d_{2} / c_{2}\right)\right\}+b_{2} \delta_{1} / 2 c_{2}, \\
a_{j}={ }_{j} c_{11}, \quad b_{i}={ }_{i} c_{13}, \quad c_{j}={ }_{j} c_{44}, \quad d_{j}={ }_{j} c_{33}, \quad 2 e_{j}={ }_{j} c_{11}-{ }_{j} c_{12}, \quad j=1,2,
\end{gathered}
$$

the ${ }_{j} c$ being the elastic constants of layer $j$. Explicitly, the low frequency, large wavelength approximation that was used in arriving at the above is $\omega=k \sqrt{P_{1}-k^{2} P_{2}}$, where $k$ denotes wavenumber.

Department of Mechanical Engineering and Applied Mechanics,

R. A. SCOTT University of Michigan,

Ann Arbor,

Michigan 48109, U.S.A.

(Received 19 June 1980)

\section{REFERENCES}

1. A. Ambardar and C. D. Ferris 1978 Journal of the Acoustical Society of America 63, 781-792. Wave propagation in a piezoelectric two-layered cylindrical shell with hexagonal symmetry: some implications for long bone.

2. R. A. SCOTT 1973 Journal of Sound and Vibration 26,321-335. Transient compressional wave propagation in a two-layered circular rod with imperfect bonding. 